\title{
Human immunodeficiency virus/acquired immunodeficiency syndrome epidemic in adolescents from a Brazilian metropolis (1978-2017)
}

\author{
Stella Regina Taquette ${ }^{[1]}$ and Nádia Cristina Pinheiro Rodrigues ${ }^{[2]}$
}

[1]. Universidade do Estado do Rio de Janeiro, Departamento de Pediatria, Rio de Janeiro, RJ, Brasil.

[2]. Universidade do Estado do Rio de Janeiro, Instituto de Medicina Social, Rio de Janeiro, RJ, Brasil.

\begin{abstract}
Introduction: Prevalence of human immunodeficiency virus among adolescents is increasing. This study aimed to analyze this current situation in Rio de Janeiro City. Methods: This was a retrospective longitudinal study using secondary data from the National System of Notifiable Diseases database of cases in adolescents aged 13-19 years. Results: There were 885 acquired immunodeficiency syndrome cases from 1978 to 2017 and 445 human immunodeficiency virus new cases from 2014 to 2017. Over time, sexually transmitted human immunodeficiency virus/acquired immunodeficiency syndrome cases increase. Conclusions: Human immunodeficiency virus/acquired immunodeficiency syndrome epidemic in adolescents requires novel prevention policies.
\end{abstract}

Keywords: HIV-AIDS. Adolescence. Prevention \& control. Health vulnerability. Public policies. Social control.

In Brazil, after a short period of incidence reduction, human immunodeficiency virus/acquired immunodeficiency syndrome (HIV/AIDS) is increasing, particularly in young men who have sex with men and pregnant women. According to the latest Epidemiological Bulletin of the Brazilian Ministry of Health on HIV/AIDS, referring to cases confirmed on June 2017, individuals between 15 and 19 years old continue to present increasing rates of AIDS incidence. According to distribution by sex, in the last 10 years, there is a reduced detection rate among women in all ages, except between 15 and 19 years old, where there was an increasing rate. It should be observed that prevalence ratio between sexes is lower in this age group compared to other age groups, from 17 men to 10 women, showing that the reduction in the current situation of epidemic feminization, observed in 2009, was lower in adolescents than other age groups. Among young individuals aged from 20 to 29 years, this ratio is 30 men for every 10 women ${ }^{1}$.

Homosexual males are predominantly exposed to HIV infection, and the number of HIV cases in homosexual males

\footnotetext{
Corresponding author: Stella Regina Taquette.

e-mail: stella.taquette@gmail.com

Orcid: 0000-0001-7388-3025

Received 23 April 2019

Accepted 24 July 2019
}

is increasing every year. A recent national HIV surveillance in a sample of 4176 men who have sex with men, performed in 12 municipalities in the five macroregions of Brazil, observed an HIV prevalence of $18.4 \%$, significantly higher than the prevalence of $12.1 \%$ observed in 2009 in a similar study. Regarding age group, this study showed that the highest prevalence increase was observed in individuals aged between 15 and 19 years, with a triple increase in prevalence ${ }^{2}$.

It is worth mentioning that AIDS epidemic in adolescents is reaching the poorest population strata ${ }^{3}$. Regarding race/color, higher AIDS prevalence was observed in the Black population than in other racial groups. In the last 10 years, there was a reduced detection rate of AIDS in the White population (21.9\%) and an increased detection rate in the Black population (35.7\%), especially among women ${ }^{1}$.

Based on the current situation of AIDS in adolescents in Brazil, this study aimed to analyze the dynamics of the epidemic between individuals aged 13-19 years in the Rio de Janeiro City since its beginning to generate information that contributes to the creation of preventive interventions in this population.

We used data collected from HIV and AIDS cases in adolescents (aged 13-19 years) from the "Brazil's Information System for Notifiable Diseases." (SINAN) and population data from census and intercensus projections of the Brazilian Institute 
of Geography and Statistics. It is worth mentioning that HIV notification has only become mandatory in Brazil since 2014. Therefore, we only have these data from 2014 . We calculated both AIDS and HIV incidence rates per 100,000 and AIDS mortality rate per 100,000 adolescents according to diagnostic year (or period of 10 years) and sex. For each individual case, we assessed sociodemographic information, type of transmission, and, in advanced cases, death.

We used chi-squared tests and Kruskal-Wallis tests to evaluate statistical difference between the number of cases by period according to sociodemographic information, type of transmission, and sexual preference.

Bayesian multilevel logistic models were used to estimate mortality AIDS odds in adolescents. Fix effects estimated in adjusted model were sex, educational level, and types of transmission (sexually transmitted, transmitted by the administration of injectable drugs, and transmitted by vertical and blood routes). In both models, we used random effects for neighborhood, diagnostic year, and Human Development Index.

We calculated generalized variance inflation factor and Hosmer and Lemeshow goodness of fit test. K-fold crossvalidation method was used to evaluate model performance on different subsets of training data $(\mathrm{k}=10)$.

The local ethics committee approved the project (CAAE $n^{\circ}$ 84670817.4.3001.5279).

There were 885 AIDS cases in adolescents (13-19 years old) from 1978 to 2017. The more recent the period, the higher the number of AIDS cases. We observed similar behavior of AIDS incidence rates, ranging from $0 / 100,000$ in the first period to $5 / 100,000$ in the last period (Table 1).

Deaths due to AIDS in adolescents decreased after 1988-1997. For females, this reduction only occurred in the last period, while for male, it occurred from 1998 to 2007. The death ratio between sexes decreased over time, ranging from 80 males per 10 females to 8 males per 10 females from the first period to the last two periods. The highest female mortality rate was observed in 1988-1997 (2/100,000), while for males, incidence rate remained constant from 1978-1987 to 1998-2007 $(1 / 100,000)$ (Table 1).

Male incidence rate increased over the whole period, ranging from $1 / 100,000$ in $1978-1987$ to $5 / 100,000$ in 2008-2017, while incidence rate in females showed a slight decrease in the last period. The ratio of AIDS cases between sexes decreased gradually until $1998-2007$, ranging from 38 to $8-9$ males per 10 females over the periods (Table 1).

Distribution of AIDS over time by sex showed that from the beginning of the epidemic, AIDS rates in adolescents increased, ranging from 0 to $8.75 / 100,000$ in 2013 for females and from 0 to 7.03/100,000 in 2014 for males. After 2013 and 2014, rates begin to decrease for females and males, respectively.

The median age of AIDS cases was 18 years old in the most periods. Regarding ethnicity, we significantly identified non-White AIDS cases. The higher the educational level, the lower the number of AIDS cases. Regarding educational levels, AIDS cases increased in the last period. The time interval between AIDS diagnosis and its notification decreased over time, ranging from 16 to 2 months in the first period and the last period, respectively (Table 2).

Comparing transmission types, we observed that the number of AIDS cases transmitted through blood and sexual routes predominated in the first and last two periods, respectively. Over time, percentage of AIDS cases transmitted through sexual and vertical routes increases, while those transmitted through blood route decreases (Table 2).

Regarding sex, for females, heterosexual females predominantly had AIDS in all periods; for males, heterosexual males predominantly had AIDS in the second period only. In the last two periods, homosexual males (men who have sex with men [MSM]) predominantly had AIDS. For both males and females with AIDS, percentage of all types of sexual transmission increases over time (Table 2).

There were 445 HIV cases in adolescents from 2014 to 2017. The highest number of HIV cases $(n=136)$ and the highest HIV rate $(20 / 100,000)$ were observed in 2015 (Table 1).

The number of HIV cases in female adolescents exceeded that of male cases in 2014. The significant ratio of HIV cases between sexes was observed in 2016, that is, 15 male cases per 10 female cases (Table 1).

The highest percentage of HIV cases for those who completed at least elementary school was observed in 2015. The time interval between HIV diagnosis and its notification decreased over time, ranging from 69 to 0 days from 2014 to 2017 (Table 2).

Comparing transmission types, we observed that in 2014-2017, the number of HIV cases transmitted sexually predominated. Until 2016, percentage of HIV cases transmitted through vertical route increases, while those transmitted through blood route decreased in the entire period (Table 2).

Adjusted analyses indicated that the odds of dying due to AIDS was two times higher in males than in females, $58 \%$ lower for those who completed elementary school, approximately $90 \%$ lower for those who were infected by sexual or vertical route, and three times higher for those infected by the administration of injectable drugs (Table 3).

Data reveal an alarming situation as the more recent the period, the higher the number of AIDS cases, particularly in individuals with lower educational level, and the number of HIV cases in the last 3 years corresponds to greater than half of all AIDS cases diagnosed since the beginning of the epidemic by 2017. The increase in prevalence of HIV/AIDS signifies a poorly controlled HIV/AIDS epidemic, although it can also show an improvement and amplification of diagnosis. This situation is in contrary to the overall reduction in AIDS cases in several countries and signifies Brazil's regression in coping with the epidemic ${ }^{4}$.

The increase of case prevalence from vertical transmission reveals late diagnosis of HIV, resulting in the improper 
TABLE 1: Distribution of morbidity and mortality by human immunodeficiency virus/acquired immunodeficiency syndrome in adolescents over time in Rio de Janeiro City, Brazil.

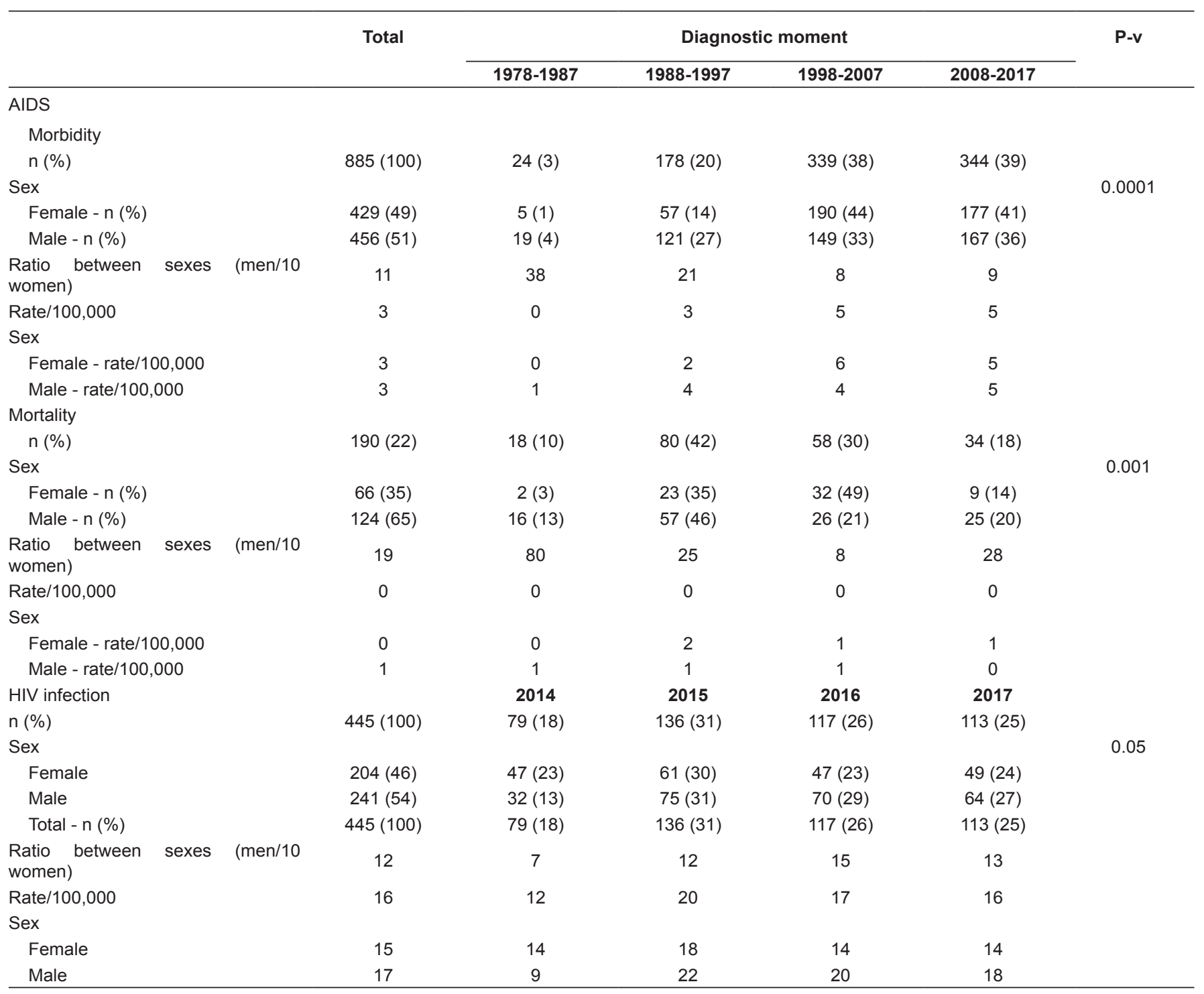

P-v, p-value; ratio between sexes, number of cases/deaths in men for each 10 cases/deaths in women.

administration of prophylaxis for vertical transmission in perinatal period and incorrect diagnosis of the disease during childhood. Such delay in HIV diagnosis in the studied population increases comorbidities of the disease and risk of HIV sexual transmission due to the lack of knowledge about seropositivity in high sexual experimentation ${ }^{5,6}$. Healthcare services have not been effective in establishing early diagnosis of HIV, and this is an essential measure to control AIDS. However, whether the establishment of late diagnosis for vertical transmission is appropriate should be properly assessed. In these cases, disease transmission may be due to unidentified sexual abuse or an error in filling out notification form.

Preventive actions for HIV/AIDS may not be accessible to youth. Today, many behavioral changes in youth brought about by digital media are observed. Conservative movements such as No-Party School ${ }^{7}$ implement imposition of silence regarding sexuality in the school environment, preventing dialogue on fundamental issues like gender, sexual orientation, sexual desire, and self-protection mechanisms. Social stigma, prejudice, and discrimination are human rights violations and barriers to disease control and prevention ${ }^{8,9}$.

The more we have a better understanding of behavioral patterns, risk practices, and social support networks, the better we can improve HIV prevention and control measures. More targeted and relevant HIV prevention programs for youth are urgently needed to prevent and control this epidemic. The increasing number of young males with HIV/AIDS is also a significant problem faced by several countries ${ }^{10}$. In the USA, $21 \%$ of all newly diagnosed HIV cases in 2016 were observed in young individuals aged 13 to 24 years, consisting of young gay and bisexual men (81\%), especially young Black/African American and Hispanic/Latino ${ }^{11}$. 
TABLE 2: Distribution of characteristics of human immunodeficiency virus/acquired immunodeficiency syndrome cases in adolescents in Rio de Janeiro City by period.

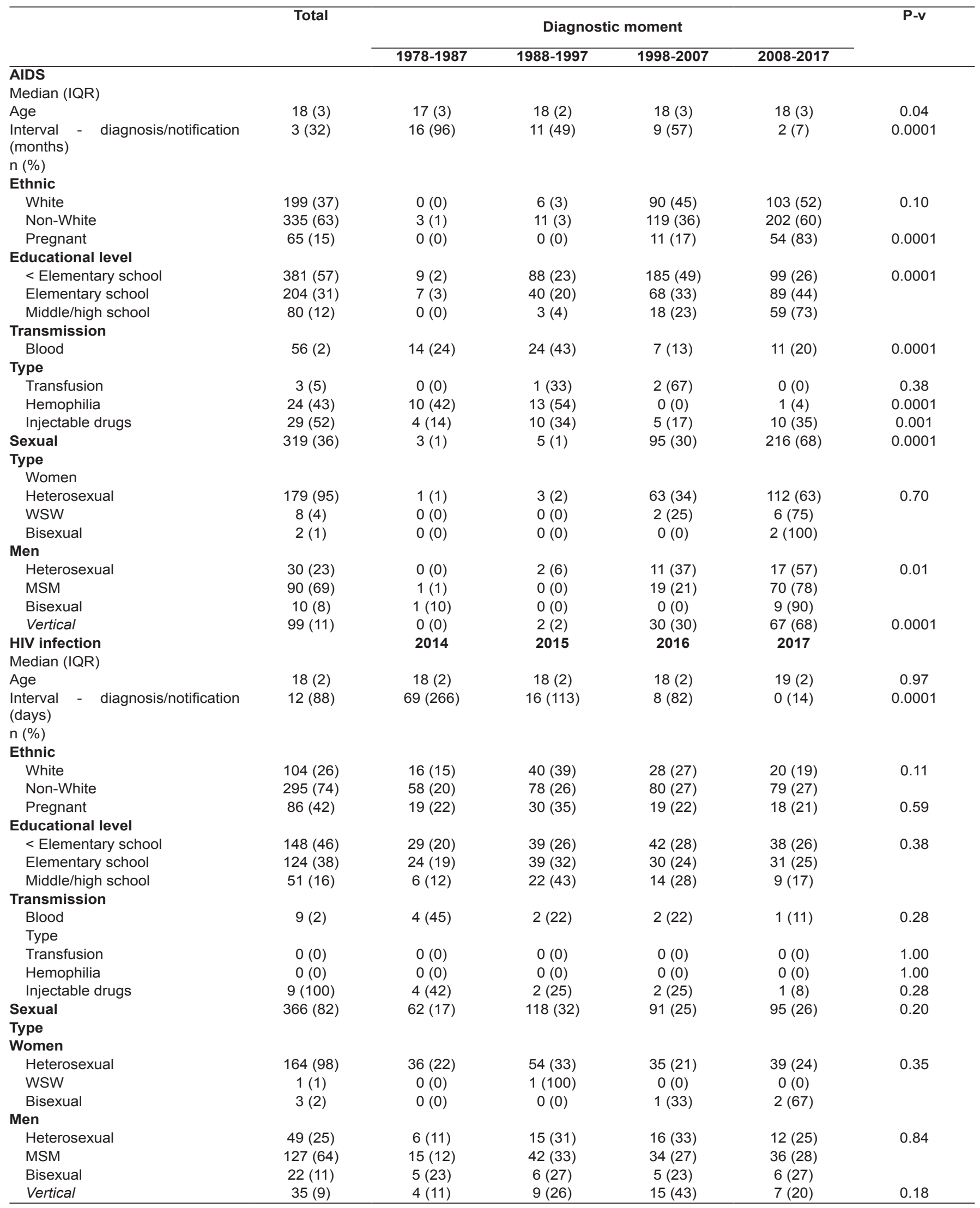

IQR, interquartile range; P-v, p-value; MSM, men who have sex with men; WSW, women who have sex with women. 
TABLE 3: Simple and adjusted models to evaluate the adolescent risk of die by acquired immunodeficiency syndrome in Rio de Janeiro City.

\begin{tabular}{|c|c|c|c|c|}
\hline & \multicolumn{2}{|c|}{ Simple models } & \multicolumn{2}{|c|}{ Adjusted model } \\
\hline & OR & $95 \% \mathrm{Cl}$ & OR & $95 \% \mathrm{Cl}$ \\
\hline \multicolumn{5}{|l|}{ Fixed effects } \\
\hline Male & 2.05 & $1.32-3.22$ & 2.12 & $1.36-3.32$ \\
\hline Age & 0.88 & $0.78-1.01$ & & \\
\hline Nonwhite race & 0.65 & $0.39-1.10$ & & \\
\hline Year & 0.86 & $0.84-0.88$ & & \\
\hline \multicolumn{5}{|l|}{ Educational level } \\
\hline$<$ Elementary school & 1.00 & & 1.00 & \\
\hline Elementary school & 0.41 & $0.24-0.67$ & 0.42 & $0.25-0.69$ \\
\hline Middle/High school & 0.27 & $0.09-0.65$ & 0.37 & $0.13-0.94$ \\
\hline \multicolumn{5}{|l|}{ Transmission } \\
\hline \multicolumn{5}{|l|}{ Blood vs. others } \\
\hline Transfusion & 1.16 & $0.07-19.88$ & & \\
\hline Hemophilia & 1.56 & $0.46-5.25$ & & \\
\hline Injectable drugs & 2.90 & $1.04-7.83$ & 3.15 & $1.29-7.58$ \\
\hline Sexual vs. others & 0.12 & $0.06-0.22$ & 0.08 & $0.04-0.13$ \\
\hline Vertical vs. others & 0.25 & $0.07-0.75$ & 0.11 & $0.03-0.33$ \\
\hline Random effects & & & Mean & \\
\hline Intercept (neighborhood) & & & 18,629 & $1,266-67,205$ \\
\hline Slope (year) & & & 18,527 & $1,242-67,031$ \\
\hline Slope (HDI) & & & 18,630 & $1,268-67,203$ \\
\hline Deviance & & & 578 & \\
\hline
\end{tabular}

OR, odds ratio; $\mathbf{C l}$, confidence interval; HDI, Human Development Index; Year, diagnostic year.

Bayesian multilevel binomial models were used to estimate the mortality AIDS odds in adolescents. Both for simple and adjusted models, the response variable was the deaths. Fix effects estimated in the adjusted model were age, sex, educational level, and types of transmission (sexually transmitted, transmitted by the administration of injectable drugs, and transmitted by vertical and blood routes). In both models, we used random effects for neighborhood, diagnostic year, and HDI.

Hence, a novel and preventive intervention is required to prevent and control this epidemic. Adolescence is a period of sexual experimentation and identification. Adolescents receive a lot of sexual information from the Web, including pornography. Today, AIDS is considered a nonfatal disease. Hence, young individuals' fear about AIDS is significantly different than to that of individuals in the past.

A better epidemiological situation of HIV/AIDS was observed in the past decade than in the current decade. Public policies in the past included significant participation of the society in preventive programs, and there were more campaigns and sexual education at schools in the past than in the present. There was an intense reduction of preventive measures aimed at these most affected populations, and government campaigns were curtailed by conservative politicians. Moreover, today, sexual freedom is significantly achieved. It is necessary to discuss issues related to sexuality and eroticism and to recognize legitimacy of all expressions of sexuality and gender. According to some individuals, condoms reduce pleasure in sexual intercourse. Pre-exposure prophylaxis is an alternative for those who cannot or do not want to use preservative consistently. Ways to achieve sexual pleasure and preventive methods are widely variable $^{12}$. Nevertheless, this type of prevention strategy is not well established in many countries, including Brazil ${ }^{13}$.

The Global Burden of Disease Health Financing Collaborator Network $^{14}$ in its study about spending on health and HIV/
AIDS in 188 countries between 1995 and 2015 shows that the spending decreased from 2013 and the lower-middleincome countries have more cases and spend less per person. The global proportion spending on prevention was $19 \%$, and in Brazil, it was $17.7 \%$. The HIV seroprevalence in youth aged 15-19 years in countries that had good extensive support programs, significant participation of the society, and improved socioeconomic status significantly decreased ${ }^{15}$.

Reduction on time interval between diagnosis and notification and decrease in the number of deaths are positively reported. Taquette and colleagues ${ }^{3}$ (2011) revealed in the previous study, with similar secondary data from the SINAN in 2009, that in approximately half of the cases, notifications occurred greater than 6 months after diagnosis. Average time between diagnosis of disease and notification was 711 days. This delay compromises the coping strategy of this disease and may show a false profile of epidemic dynamic. Another point worth mentioning is reduction of deaths as a result of universal access to treatment and compulsory licensing of efavirenz in 2007 in Brazil.

This study was conducted using secondary individual notified data of HIV/AIDS. However, the SINAN does not totally show the real epidemic situation because of delay in notification and undernotification. This is one of the limitations of the study. Another limitation is the possibility of filling out notification forms incorrectly. A third limitation may be failure 
in identifying HIV transmission type, as pointed out in the high prevalence of vertical transmission in the studied group. Additionally, the study is restricted to Rio de Janeiro City only, which does not reflect the current HIV/AIDS situation in other regions of the country. However, we believe that it offers valuable alternative interventions to public policies to fight HIV/ AIDS epidemic in adolescents and young individuals.

\section{ACKNOWLEDGMENTS}

We offer our thanks to the Coordination of Transmissible Diseases of the Municipal Health Secretariat of Rio de Janeiro for the availability of the SINAN-AIDS databases studied.

\section{Conflict of interest}

The authors declare that there is no conflict of interest.

\section{REFERENCES}

1. Ministério da Saúde (MS). Secretaria de Vigilância em Saúde. Boletim epidemiológico HIV-Aids. Brasília, DF: Ministério da Saúde; 2017, 64p.

2. Kerr L, Kendall C, Guimarães MKC, Mota RS, Veras MA, Dourado I, et al. HIV prevalence among men who have sex with men in Brazil: results of the 2nd national survey using respondent-driven sampling. Medicine (Baltimore). 2018;97:S9-S15.

3. Taquette SR, Matos HJ, Rodrigues AO, Bortolotti LR, Amorin E. A epidemia de AIDS em adolescentes de 13 a 19 anos, no município do Rio de Janeiro: descrição espaço-temporal. Rev Soc Bras Med Trop. 2011;44(4):467-70.

4. United Nations Programme on HIV/AIDS (UNAIDS). Prevention Gap Report. UNAIDS; 2016. Available from: http://www.unaids. org/sites/default/files/media_asset/2016-prevention-gap-report en.pdf

5. Moreira-Silva SF, Zandonade E, Frauches DO, Machado EA, Lopes LIA, Duque LL, et al. Comorbidities in Children and Adolescents with AIDS Acquired by HIV Vertical Transmission in Vitória, Brazil. Plos One. 2013;8(12):e82027.

6. Dias CF, Moreira-Silva SF, Reis MA, Patrício LR, Gavioli CFB, Miranda AE. Late diagnosis and HIV infection in children attending a service of specialized care for pediatric AIDS in Brazil. Rev Soc Bras Med Trop. 2014;47(1):93-6.

7. Frigotto G (org). Escola "Sem" Partido. Esfinge que ameaça a educação e a sociedade brasileira. Rio de Janeiro: UERJ, LPP; 2017. 144p.

8. Malta M, Beyrer C. The HIV epidemic and human rights violations in Brazil. J Int AIDS Soc. 2013;16(1):18817.

9. Stahlman S, Beyrer C, Sullivan PS, Mayer KH, Baral SD Engagement of gay men and other men who have sex with men (MSM) in the response to HIV: a critical step in achieving an AIDSfree generation. AIDS Behav. 2016;20(Suppl 3):330-40.

10. Huerga H, Van Cutsem G, Ben Farhat J, Puren A, Bouhenia M, Wiesner L, et al. Progress towards the UNAIDS 90-90-90 goals by age and gender in a rural area of KwaZulu-Natal, South Africa: a household-based community cross-sectional survey. BMC Public Health. 2018;18(1):303.

11. Centers for Disease Control and Prevention (CDC) HIV among Youth [Acessed 03 Oct 2018]. Available from: www.cdc.gov/hiv/ group/age/youth/index.html

12. The Lancet Infectious Diseases. Treatment as prevention for HIV. Lancet Infect Dis. 2011;11(9):651.

13. Beyrer C, Baral SD, Griensven FV, Goodreau SM, Chariyalertsak $\mathrm{S}$, Wirtz AL, et al. Global epidemiology of HIV infection in men who have sex with men. Lancet. 2012;380(9839):367-77.

14. Global Burden of Disease Health Financing Collaborator Network. Spending on health and HIV/AIDS: domestic health spending and development assistance in 188 countries, 1995-2015. The lancet. 2018;391(10132):1799-829.

15. Kornilova MS, Batluk JV, Yorick RV, Baughman AL, Hillis SD, Vitek CR. Decline in HIV seroprevalence in street youth 2006-2012, St. Petersburg, Russia: moving toward an AIDS-free generation. Int J STD AIDS. 2017;28(4):345-56.

\section{Erratum}

\section{Revista da Sociedade Brasileira de Medicina Tropical/Journal of the Brazilian Society of Tropical Medicine}

Title: Human immunodeficiency virus/acquired immunodeficiency syndrome epidemic

in adolescents from a Brazilian metropolis (1978-2017)

Vol.:53:e20190193: 2020 - doi: 10.1590/0037-8682-0193-2019 - AUTHOR

\section{Nádia Cristina Pereira Rodrigues}

Should read:

\section{Nádia Cristina Pinheiro Rodrigues}

